\title{
THE USE OF D-MODULES TO STUDY EXPONENTIAL POLYNOMIALS
}

\author{
C. A. BERENSTEIN \\ Mathematics Department $\mathcal{G}$ Institute of Systems Research, University of Maryland \\ College Park, Maryland 20742, U.S.A. \\ A. YGER \\ Département de Mathématiques, Université de Bordeaux I \\ 33405 Talence, France
}

\begin{abstract}
This is a summary of recent work where we introduced a class of $\mathcal{D}$-modules adapted to study ideals generated by exponential polynomials.

0. Introduction. This is an expanded version of the lecture given by the first author at the Banach Center, during the workshop on residues, November 1992. We introduce a new method to study ideals generated by exponential polynomials, inspired by the theory on $\mathcal{D}$-modules $[16,17,18]$. Detailed proofs of some of the statements of this paper can be found in [14]. We take the opportunity to thank Professors Jakóbczak, Pleśniak, and Aizenberg for their hospitality.

Let us recall that an exponential polynomial $f$ of $n$ complex variables with frequencies in a finitely generated subgroup $\Gamma$ of $\mathbb{C}^{n}$ is a function of the form

$$
f\left(z_{1}, \ldots, z_{n}\right)=f(z)=\sum_{\gamma \in \Gamma} p_{\gamma}(z) \exp (\gamma \cdot z),
$$

where the sum is finite, the $p_{\gamma}$ are polynomials, and $\gamma \cdot z=\gamma_{1} z_{1}+\ldots+\gamma_{n} z_{n}$. Such a function belongs to the algebra $A_{\phi}\left(\mathbb{C}^{n}\right)$ of entire functions $F$ satisfying the growth condition:

$$
\exists C>0 \quad|F(z)| \leq C \exp (C \phi(z)),
$$

1991 Mathematics Subject Classification: 32A15, 32A25, 34K05, 34K35, 32S40.

Research of the first author partially supported by the NSF grants DMS-9000619 and CDR8803012 .

Research of the second author partially supported by the NSF grant DMS-9000619.

The paper is in final form and no version of it will be published elsewhere.
\end{abstract}


where the weight $\phi$ can be taken as $|z|$, the Euclidean norm of $z$, or even better, if we choose a system $\gamma^{1}, \ldots, \gamma^{N}$, of $\mathbb{Q}$-linearly independent generators of $\Gamma$, as

$$
\phi(z)=\max \left(\left|\Re\left(\gamma^{j} \cdot z\right)\right|: j=1, \ldots, N\right)+\log \left(1+|z|^{2}\right),
$$

where $\Re z$ denotes the real part of the complex number $z$. In the case that $\Gamma \subset$ $i \mathbb{R}^{n}$, the exponential polynomials are just the Fourier transforms of distributions supported by finitely many points in the lattice $-i \Gamma$, and $A_{\phi}$ is a subalgebra of the Paley-Wiener algebra $\widehat{\mathcal{E}^{\prime}\left(\mathbb{R}^{n}\right)}$ of Fourier transforms of distributions of compact support. In this case, the exponential polynomials are the Fourier transforms of linear difference-differential operators with constant coefficients in $\mathbb{R}^{n}$ (sometimes called transfer function, characteristic function, symbol, according to the area of mathematics or engineering involved in the problem).

The motivation of our work is the study of the solutions of arbitrary systems of difference-differential equations. When the dimension $n=1$, the spectral synthesis theorem [24] guarantees that every $C^{\infty}$ (or distribution) solution of such a system (more generally, any system of convolution equations of compact support) belongs to the closure of the simplest solutions of the system, namely the exponential polynomial solutions that have frequencies in the collection of common zeros of the transfer functions of the original equations. It is less well-known that the spectral synthesis does not hold for arbitrary systems of convolution equations as soon as $n \geq 2$, equivalently, not all ideals in the Paley-Wiener algebra are localizable [20]. If an ideal is generated by polynomials then, it has been proved by Ehrenpreis and Malgrange, that it is always localizable [19, 21]. The only fairly general criterion to ensure localizability of a finitely generated ideal $I$ is to verify that the generators form a slowly decreasing sequence in the sense of [4]. Among other requirements, the generators must define a complete intersection. The slowly decreasing condition is not too easy to check, especially when the variety $V$ of common zeros of the generators is not discrete. The only general example given in [4] of a slowly decreasing sequence of exponential polynomials is the following. Let $P_{1}, \ldots, P_{n}$ be polynomials defining a discrete (hence, finite) variety in $\mathbb{C}^{n}$ and $k \leq n$, then the sequence of functions

$$
f_{j}(z)=P_{j}\left(e^{i z_{1}}, \ldots, e^{i z_{k}}, z_{k+1}, \ldots, z_{n}\right) \quad(1 \leq j \leq n)
$$

is slowly decreasing, with discrete (but infinite) variety $V$ of common zeros.

For these reasons, in one of our previous papers [7] we considered the case of finitely generated ideals of exponential polynomials with frequencies in a group $\Gamma$ of rank exactly $n$ and assumed that $V$ was discrete. Even when $\Gamma=i \mathbb{Z}^{n}$, we could not find a general criterion for localizability of the ideals generated by such exponential polynomials. Part of the problem is of an arithmetic nature, namely localizability may depend not only on the geometry of $V$ and $\Gamma$, but also on the diophantine approximations of the coefficients of the generators of $I$. For example, the ideal generated by $\cos \left(z_{1}\right), \cos \left(z_{2}\right), z_{2}-\alpha z_{1}$ is localizable if and only if $\alpha$ is not a Liouville number. As we pointed out in [8], there is a deep relationship 
between the localizability issue and the following conjecture of Ehrenpreis on the zeros of exponential polynomials of a single variable with algebraic coefficients and frequencies.

Conjecture 0.1. Let $a_{1}, \ldots, a_{m} \in \overline{\mathbb{Q}}[z]$ be non-zero, $\alpha_{1}, \ldots, \alpha_{m}$ be distinct numbers in $\overline{\mathbb{Q}} \cap \mathbb{R}$, and $f$ be the exponential polynomial of a single variable given by

$$
f(z)=\sum_{j=1}^{m} a_{j}(z) e^{i \alpha_{j} z} .
$$

In that case, there exist constants $\varepsilon>0, N \geq 0$ such that if $z_{1}, z_{2}$ are two different zeros of $f$ then

$$
\left|z_{1}-z_{2}\right| \geq \frac{\varepsilon}{\left(\left|z_{1}\right|+\left|z_{2}\right|\right)^{N}}
$$

In our more recent paper [14] we consider a situation that is fairly different from that of [7]. Namely, the group $\Gamma$ has very low rank, either one or two, and the variety $V$ might not be discrete or complete intersection. Using a convenient generalization of the Weyl algebra, we have obtained some results that are very simple to state. For instance, if $\operatorname{rank}(\Gamma)=1$, any system of exponential polynomials defining a complete intersection generates a localizable ideal in the space $A_{\phi}$. Another example of localizability occurs when the generators are of the type (1) and define a non-discrete complete intersection. We have also studied problems related to global versions of the Nullstellensatz and of the Briançon-Skoda theorem, which could be useful when solving the ubiquitous Bezout identity for exponential polynomials without common zeros. The solution of the Ehrenpreis conjecture mentioned earlier, is precisely equivalent to solving in general the Bezout identity.

The leitmotiv of our approach is to relate the division problems implicit in the previous questions, to the study of the analytic continuation, as a function of $\lambda_{1}, \ldots, \lambda_{m}$, of the distribution

$$
z \mapsto\left|f_{1}(z)\right|^{\lambda_{1}} \ldots\left|f_{m}(z)\right|^{\lambda_{m}}
$$

for exponential polynomials $f_{j}$. In fact, the residues of this distribution-valued meromorphic functions give the information needed to solve the Bezout equation and other division problems. This idea originated in our previous work about residue currents [2] and their applications to the effective solvability of the polynomial membership problem $[10,11]$. The reader can consult our forthcoming monograph [3], for an overview of the ideas we use. The theory of $\mathcal{D}$-modules, as introduced by J. Bernstein [16], was precisely formulated to obtain an explicit form of the analytic continuation in $\lambda$ of the distribution $|P(z)|^{\lambda}$ when $P$ is a polynomial. Bernstein's results were extended by Björk to the holomorphic setting in [17]. The main point used in Bernstein's work is that one deals with holonomic $\mathcal{D}$-modules, the new difficulty that arises for exponential polynomials is that the $\mathcal{D}$-modules one needs to consider are not holonomic. 
1. A generalization of the Weyl algebra. The ideas we present in this section are clearly related to those about the Weyl algebra found in [17, Chapter 1], to which we refer for further developments.

We denote by $\mathbb{N}$ the set of non-negative integers. For an index $\alpha \in \mathbb{N}^{n}$, its length $|\alpha|=\alpha_{1}+\ldots+\alpha_{n}$. We also let $\mathbb{K}$ be a field of characteristic zero, $n$ and $m$ two positive integers, we define an extension $E_{n, m}(\mathbb{K})$ of the Weyl algebra $A_{n}(\mathbb{K})$. It is an algebra of operators acting on the algebra of polynomials in $n+m$ variables over $\mathbb{K}$ as follows.

Consider the polynomial algebra $\mathbb{K}\left[x_{1}, \ldots, x_{n}, y_{1}, \ldots, y_{m}\right]$ and derivations $D_{1}, \ldots, D_{n}$ on this algebra such that

$$
\begin{gathered}
D_{i} x_{j}=\delta_{i j} \quad(i, j=1, \ldots, n), \\
D_{i} y_{j}=\delta_{i j} y_{j} \quad(i=1, \ldots, n ; j=1, \ldots, m) .
\end{gathered}
$$

The algebra $E_{n, m}(\mathbb{K})$ is the algebra of operators on $\mathbb{K}\left[x_{1}, \ldots, x_{n}, y_{1}, \ldots, y_{m}\right]$ generated by $X_{1}, \ldots, X_{n}, Y_{1}, \ldots, Y_{m}, D_{1}, \ldots, D_{n}$, where $X_{i}$ (resp. $Y_{j}$ ) is the operator of multiplication by $x_{i}$ (resp. $y_{j}$ ). It is a Lie algebra, with the usual definition of the Lie bracket $[.,$.$] in terms of the composition of operators, i.e.,$

$$
[P, Q]=P \circ Q-Q \circ P .
$$

The Lie bracket satisfies the following commutator relations

$$
\begin{gathered}
{\left[X_{i}, X_{j}\right]=\left[Y_{i}, Y_{j}\right]=\left[X_{i}, Y_{j}\right]=\left[D_{i}, D_{j}\right]=0 ;} \\
{\left[X_{i}, D_{j}\right]=-\delta_{i j} ; \quad\left[Y_{i}, D_{j}\right]=-\delta_{i j} Y_{i} .}
\end{gathered}
$$

We note that for $m=0$ our algebra coincides with the Weyl algebra. Every element $P$ of $E_{n, m}(\mathbb{K})$ can be written in a unique way as a finite sum

$$
P=\sum_{\alpha, \beta, \gamma} c_{\alpha, \beta, \gamma} X^{\alpha} Y^{\beta} D^{\gamma}
$$

$c_{\alpha, \beta, \gamma} \in \mathbb{K}, \alpha, \gamma \in \mathbb{N}^{n}, \beta \in \mathbb{N}^{m}$. The integer $\max \left(|\alpha|+|\beta|+|\gamma|: c_{\alpha, \beta, \gamma} \neq 0\right)$ is denoted $\operatorname{deg} P$. It is convenient to introduce the operators $\operatorname{ad}(Q)$ acting on $E_{n, m}(\mathbb{K})$ by $\operatorname{ad}(Q)(P):=[P, Q]$. One has the following simple calculus rules.

LEMMA 1.1. For any integers $a, b \geq 0,1 \leq k \leq n$, we have

$$
\left[D_{k}, X_{k}^{a} Y_{k}^{b}\right]=a X_{k}^{a-1} Y_{k}^{b}+b X_{k}^{a} Y_{k}^{b} .
$$

Corollary 1.1. Let $P(X, Y)=\sum_{k=0}^{M} X_{1}^{k} P_{k}\left(X^{\prime}, Y\right)=\sum_{l=0}^{N} Y_{1}^{l} Q_{l}\left(X, Y^{\prime}\right)$, where $X=\left(X_{1}, X^{\prime}\right), Y=\left(Y_{1}, Y^{\prime}\right)$. Then

$\left[D_{1}, P\right]=\sum_{l=0}^{N} Y_{1}^{l}\left(\frac{\partial Q_{l}}{\partial X_{1}}+l Q_{l}\right)=X_{1}^{M} Y_{1} \frac{\partial P_{M}}{\partial Y_{1}}+\sum_{k=0}^{M-1} X_{1}^{k}\left\{(k+1) P_{k+1}+Y_{1} \frac{\partial P_{k}}{\partial Y_{1}}\right\}$.

These calculus rules are necessary to study the natural filtration $\mathcal{E}_{v}$, defined on $E_{n, m}(\mathbb{K})$ by

$$
\mathcal{E}_{v}:=\left\{P \in E_{n, m}(\mathbb{K}): \operatorname{deg} P \leq v\right\}
$$


It is a $\mathbb{K}$-vector space of dimension $\left(\begin{array}{c}2 n+m+v \\ v\end{array}\right) \approx v^{2 n+m}$. As usual [17], one introduces the graded algebra $\operatorname{gr}\left(E_{n, m}(\mathbb{K})\right)$ by

$$
\operatorname{gr}\left(E_{n, m}(\mathbb{K})\right):=\mathcal{E}_{0} \oplus \mathcal{E}_{1} / \mathcal{E}_{0} \oplus \ldots
$$

The above lemma can be used to show this algebra is commutative. Moreover, $\operatorname{gr}\left(E_{n, m}(\mathbb{K})\right)$ is isomorphic to a polynomial ring in $2 n+m$ variables.

Let $M$ be a (left) $E_{n, m}(\mathbb{K})$-module and $\Gamma_{v}$ a filtration of $M$, i.e., an increasing family of finite dimensional $\mathbb{K}$-vector spaces $\Gamma_{v}$ such that

(i) $\bigcup_{v>0} \Gamma_{v}=M$;

(ii) $X_{i} \bar{\Gamma}_{v} \subseteq \Gamma_{v+1}, Y_{i} \Gamma_{v} \subseteq \Gamma_{v+1}$, and $D_{i} \Gamma_{v} \subseteq \Gamma_{v+1}$.

Let $\Gamma(v):=\Gamma_{v} / \Gamma_{v-1}$ and define $\operatorname{gr}(M)$ by

$$
\operatorname{gr}(M):=\Gamma_{0} \oplus \Gamma_{1} / \Gamma_{0} \oplus \ldots=\Gamma(0) \oplus \Gamma(1) \oplus \ldots
$$

Due to property (ii), this graded module is a module over $\operatorname{gr}\left(E_{n, m}(\mathbb{K})\right)$. One says the filtration is a good filtration if $\operatorname{gr}(M)$ is of finite type over $\operatorname{gr}\left(E_{n, m}(\mathbb{K})\right)$. For instance, if $M$ is finitely generated over $E_{n, m}(\mathbb{K})$ by $a_{1}, \ldots, a_{r}$ and we choose $\Gamma_{v}:=\mathcal{E}_{v} a_{1}+\ldots+\mathcal{E}_{v} a_{r}$, then we have a good filtration. As in [17, Lemma 3.4], one can prove the following lemma.

LEMmA 1.2. Let $\left(\Gamma_{v}\right)_{v},\left(\Omega_{v}\right)_{v}$ be two filtrations of an $E_{n, m}(\mathbb{K})$ module $M$, and assume that $\left(\Gamma_{v}\right)_{v}$ is a good filtration. Then there is an integer $w$ such that $\Gamma_{v} \subseteq \Omega_{v+w}$ for all $v \geq 0$.

If $\operatorname{gr}(M)$ is of finite type over $\operatorname{gr}\left(E_{n, m}(\mathbb{K})\right)$, there is a Hilbert polynomial $H \in \mathbb{Q}[t]$ such that for all $v \gg 1$

$$
H(v)=\operatorname{dim}_{\mathbb{K}} \Gamma_{v}
$$

(see [17, Theorem 3.1]). As a consequence of the last lemma, the degree and the leading coefficient of $H$ do not depend on the choice of the good filtration $\left(\Gamma_{v}\right)_{v}$. The degree $d$ of $H$ is called the dimension $d(M)$ of $\operatorname{gr}(M)$ and the multiplicity $e(M)$ of $\operatorname{gr}(M)$ is the leading term of $H$ times $d$ !. In the case $m=0$, i.e., for the Weyl algebra $A_{n}(\mathbb{K})$ one has the fundamental theorem of J. Bernstein that asserts that, for any non-trivial $A_{n}(\mathbb{K})$-module $M$ so that $\operatorname{gr}(M)$ is of finite type,

$$
d(M) \geq n \text {. }
$$

An $A_{n}(\mathbb{K})$-module $M$ such that $d(M)=n$ is said to be holonomic.

One of the applications of the concept of holonomic modules is the existence of the Bernstein-Sato functional equations [17, 25, 23], i.e., given polynomials $f_{1}, \ldots, f_{q}$ in $\mathbb{K}\left[x_{1}, \ldots, x_{n}\right]$ there are differential operators $Q_{j}$ in $A_{n}(\mathbb{K}[\lambda])$, with $\lambda=\left(\lambda_{1}, \ldots, \lambda_{q}\right)$, and a non-zero polynomial $b \in \mathbb{K}[\lambda]$ such that the formal relations

$$
Q_{j}\left(f_{1}^{\lambda_{1}} \ldots f_{j}^{\lambda_{j}+1} \ldots f^{\lambda_{q}}\right)=b(\lambda) f_{1}^{\lambda_{1}} \ldots f^{\lambda_{q}} \quad(j=1, \ldots, q)
$$

hold. 
We are interested in the following $E_{n, m}(\mathbb{K})$-modules, with $m \leq n$. Consider exponential polynomials $P_{1}, \ldots, P_{q}$ of $n$ variables with positive integral frequencies and coefficients in a subfield $\mathbb{K}$ of $\mathbb{C}$, that is, finite sums

$$
P_{j}(x)=\sum_{k \in \mathbb{N}^{m}} c_{j, k}(x) e^{k \cdot x},
$$

with $c_{j, k} \in \mathbb{K}[x], j=1, \ldots, q$. We consider a new field $\mathbb{K}(\lambda)=\mathbb{K}\left(\lambda_{1}, \ldots, \lambda_{q}\right)$ obtained from $\mathbb{K}$ by adjoining $q$ indeterminates, and define the module $M$ freely generated by a single generator denoted $\mathcal{P}^{\lambda}=\mathcal{P}_{1}^{\lambda_{1}} \ldots \mathcal{P}_{q}^{\lambda_{q}}$, namely,

(3) $\quad M=M\left(P_{1}, \ldots, P_{q}\right):=\mathbb{K}(\lambda)\left[x_{1}, \ldots, x_{n}, e^{x_{1}}, \ldots, e^{x_{m}}\right]\left[1 / P_{1}, \ldots, 1 / P_{q}\right] \mathcal{P}^{\lambda}$,

where, to pick up the earlier notation, $X_{i}$ (resp., $Y_{j}$ ) operates as multiplication by $x_{i}$ (resp., by $e^{x_{j}}$ ) and $D_{j}$ acts as the differential operator $\nabla_{j}$, defined by

$$
\nabla_{j}\left(A \mathcal{P}^{\lambda}\right):=\left(\frac{\partial A}{\partial x_{j}}+A \sum_{k=1}^{q} \frac{\lambda_{k}}{P_{k}} \frac{\partial P_{k}}{\partial x_{j}}\right) \mathcal{P}^{\lambda} .
$$

The natural filtration of $M$ is

$$
\Gamma_{v}:=\left\{\frac{R\left(\lambda, x, e^{x}\right)}{\left(P_{1} \ldots P_{q}\right)^{v}} \mathcal{P}^{\lambda}: R \in \mathbb{K}(\lambda)\left[x, e^{x}\right], \operatorname{deg}_{x, e^{x}} R \leq v d_{0}\right\},
$$

where $d_{0}:=1+\operatorname{deg}_{x, e^{x}}\left(P_{1} \ldots P_{q}\right)$. This is a good filtration and

$$
\operatorname{dim}_{\mathbb{K}(\lambda)} \Gamma_{v}=\left(\begin{array}{c}
n+m+v d_{0} \\
v d_{0}
\end{array}\right) .
$$

Hence,

$$
d(M)=n+m, \quad e(M)=d_{0}^{n+m} .
$$

It is natural to ask whether for every non-trivial $E_{n, m}(\mathbb{K})$-module (or $E_{n, m}(\mathbb{K}(\lambda))$-module) with $m \leq n$, one has $d(M) \geq n+m$. Or, at least, to give conditions that ensure this inequality occurs. One can give examples showing that this may depend on the choice of field $\mathbb{K}$.

In the case $m=1$, as a substitute for Bernstein's theorem we have the following result.

Proposition 1.1. Let $M$ be a finitely generated $E_{n, 1}(\mathbb{K})$-module, then, either $d(M) \geq n+1$ or for every element $m_{0} \in M \backslash\{0\}$ there exist two non-zero polynomials $A, B \in \mathbb{K}[s]$, and $t \in \mathbb{N}$ such that

$$
Y_{1}^{t} A\left(X_{1}\right) m_{0}=B\left(Y_{1}\right) m_{0}=0 .
$$

As an application of Proposition 1.1 to the module

$$
M\left(P_{1}, \ldots, P_{q}\right)=\mathbb{K}(\lambda)\left[x_{1}, \ldots, x_{n}, e^{x_{1}}\right]\left[1 / P_{1}, \ldots, 1 / P_{q}\right] \mathcal{P}^{\lambda}
$$

defined by equation (2), where $P_{j} \in \mathbb{K}\left[x_{1}, \ldots, x_{n}, e^{x_{1}}\right], \mathbb{K}$ a subfield of $\mathbb{C}$, one can prove the following proposition. 
Proposition 1.2. There are two non-zero polynomials $A_{1}, A_{2}$ of a single variable $s$, with coefficients in $\mathbb{K}[\lambda], \lambda=\left(\lambda_{1}, \ldots, \lambda_{q}\right)$, and $2 q$ linear differential operators, $Q_{i, j}(i=1,2 ; j=1, \ldots, q)$, with coefficients belonging to $\mathbb{K}\left[\lambda, x, e^{x_{1}}, e^{-x_{1}}\right]$, such that for every $j$,

$$
\begin{aligned}
& A_{1}\left(\lambda, x_{1}\right) \mathcal{P}^{\lambda}=Q_{1, j}\left(\lambda, x, e^{x_{1}}, e^{-x_{1}}, \partial / \partial x\right) P_{j} \mathcal{P}^{\lambda}, \\
& A_{2}\left(\lambda, e^{x_{1}}\right) \mathcal{P}^{\lambda}=Q_{2, j}\left(\lambda, x, e^{x_{1}}, e^{-x_{1}}, \partial / \partial x\right) P_{j} \mathcal{P}^{\lambda} .
\end{aligned}
$$

( To simplify the notation we have written $\partial / \partial x$ to denote $\left(\partial / \partial x_{1}, \ldots, \partial / \partial x_{n}\right)$.)

2. Functional equations. As explained in [3], to study Bézout identities, division problems, and the like, one needs to determine the principal part of the Laurent development of $|f|^{2 \lambda}$ for $\lambda=-k, k \in \mathbb{N}$, where $f$ is an exponential polynomial. The reason for this need will become clear later on. The following lemma, a consequence of Proposition 1.2, provides some of this information.

LEMmA 2.1. Let $f$ be an exponential polynomial in $E_{n, 1}(\mathbb{K}), k \in \mathbb{N}$, there is an integer $q \in \mathbb{N}$ such that for any $N \in \mathbb{N}$ one can find a non-zero polynomial $R_{N} \in \mathbb{K}\left[x_{1}\right]$ and a functional equation of the form

$$
(\lambda+k)^{q} R_{N}|f|^{2 \lambda}=Q_{k, N}\left(|f|^{2 \lambda} f^{k+1}\right)+(\lambda+k)^{q+N} v_{N}|f|^{2 \lambda},
$$

where $v_{N} \in \mathbb{K}\left[\lambda, x_{1}\right]$ and $Q_{k, N}$ is a linear differential operator with coefficients in $\mathbb{K}\left[\lambda, x, e^{x_{1}}, e^{-x_{1}}\right]$.

More generally, one can obtain relations of the form

$$
(\lambda+k)^{\tilde{q}} S_{N}\left(e^{x_{1}}\right)|f|^{2 \lambda}=\tilde{Q}_{k, N}\left(|f|^{2 \lambda} f^{2 k+1}\right)+(\lambda+k)^{\tilde{q}+N} \tilde{v}_{N}|f|^{2 \lambda},
$$

where $\tilde{v}_{N} \in \mathbb{K}\left[\lambda, e^{x_{1}}\right], S_{N}(t)=S_{N, k}(t) \in \mathbb{K}[t]$, and $\tilde{Q}_{k, N}$ is a differential operator with the same properties as $Q_{k, N}$.

The way one uses these relations is the following. One knows a priori [1] that, in a neighborhood of $\lambda=-k$, the distribution-valued meromorphic function $|f|^{2 \lambda}$ has the Laurent expansion

$$
|f|^{2 \lambda}=\sum_{j=-2 n}^{\infty} a_{k, j}(\lambda+k)^{j},
$$

with $a_{k, j} \in \mathcal{D}^{\prime}\left(\mathbb{C}^{n}\right)$. The previous lemma allows us to compute explicitly the products $R_{N}\left(x_{1}\right) a_{k, j}, S_{N}\left(e^{x_{1}}\right) a_{k, j}$, for $-2 n \leq j \leq 0$, if we let $N=2 n+1$. Namely, the polynomial $v_{N}$ in the statement of Lemma 2.1 can be expanded in powers of $\lambda+k$, i.e.,

$$
v_{N}\left(\lambda, x_{1}\right)=\sum_{l=0}^{m} v_{N, l}\left(x_{1}\right)(\lambda+k)^{l} .
$$


Let $\varphi \in \mathcal{D}^{\prime}\left(\mathbb{C}^{n}\right)$, then

$$
\begin{aligned}
(\lambda+k)^{q}\left\langle|f|^{2 \lambda}, R_{N}\left(x_{1}\right) \varphi\right\rangle= & \sum_{j=-2 n}^{\infty}\left\langle a_{k, j}, R_{N} \varphi\right\rangle(\lambda+k)^{q+j} \\
= & \left\langle Q_{k, N}(\lambda)\left(|f|^{2 \lambda} f^{2 k+1}\right), \varphi\right\rangle \\
& +\sum_{j=-2 n}^{\infty}\left\langle a_{k, j}, v_{N} \varphi\right\rangle(\lambda+k)^{q+N+j} \\
= & \left\langle|f|^{2 \lambda} f^{2 k+1}, Q_{k, N}^{\prime}(\lambda) \varphi\right\rangle \\
& +\sum_{j, l}\left\langle a_{k, j}, v_{N, l} \varphi\right\rangle(\lambda+k)^{q+N+j+l},
\end{aligned}
$$

where $Q_{k, N}^{\prime}$ is the adjoint operator of $Q_{k, N}$ (obtained by integration by parts).

The first term of the last sum is holomorphic at $\lambda=-k$, and the series only contains powers of $\lambda+k$ bigger than or equal to $q+1$, due to the choice of $N$. Thus, the distribution-valued function $(\lambda+k)^{q} R_{N}\left(x_{1}\right)|f|^{2 \lambda}$ is holomorphic in a neighboorhood of $\lambda=-k$. Moreover, if we denote

$$
(\lambda+k)^{q} R_{N}\left(x_{1}\right)|f|^{2 \lambda}=\sum_{h=0}^{\infty} b_{k, h}(\lambda+k)^{h}
$$

its Taylor development, then, for $0 \leq h \leq q$, the distributions $b_{k, h}$ are given by

$$
\left\langle b_{k, h}, \varphi\right\rangle=\frac{1}{2 \pi i} \int_{|\lambda+k|=\varepsilon} \int_{\mathbb{C}^{n}}|f(x)|^{2 \lambda} f(x)^{2 k+1} Q_{k, N}^{\prime}(\lambda)(\varphi(x)) d x \frac{d \lambda}{(\lambda+k)^{h+1}},
$$

where $\varepsilon>0$ is chosen sufficiently small so that on a neighborhood of $\operatorname{supp}(\varphi)$, the function $x \mapsto|f(x)|^{-2 \varepsilon}$ is integrable.

We can rewrite the last integral as

$$
\begin{aligned}
& \frac{1}{2 \pi i} \int_{|\lambda+k|=\varepsilon} \int_{\mathbb{C}^{n}}|f(x)|^{2(\lambda+k)} f(x)(f(x) / \overline{f(x)})^{k} Q_{k, N}^{\prime}(\lambda)(\varphi(x)) d x \frac{d \lambda}{(\lambda+k)^{h+1}} \\
& \quad=\sum_{j=0}^{\infty} \frac{1}{j !} \frac{1}{2 \pi i} \int_{|\lambda+k|=\varepsilon} \int_{\mathbb{C}^{n}}\left(\log |f|^{2}\right)^{j} f(f / \bar{f})^{k} Q_{k, N}^{\prime}(\lambda)(\varphi(x)) d x(\lambda+k)^{j-h-1} d \lambda,
\end{aligned}
$$

which shows that the terms $\left\langle b_{k, h}, \varphi\right\rangle$ are linear combinations of integrals of the form

$$
\int_{\mathbb{C}^{n}}\left(\log |f|^{2}\right)^{j} f(f / \bar{f})^{k} Q_{\iota}(\varphi) d x
$$

where $j \in \mathbb{N}$ (in fact, $0 \leq j \leq h+1$ ) and the $Q_{\iota}$ are differential operators with coefficients in $\mathbb{K}\left[x, e^{x_{1}}, e^{-x_{1}}\right]$. Note that the term $(f / \bar{f})^{k}$ is bounded, and the same holds locally for $f\left(\log |f|^{2}\right)^{j}$. 
Since $R_{N}$ really depends now only on $k$ we shall denote it $\mathcal{R}_{k}$ from now on. Therefore, from (8) and (11) we obtain

$$
\begin{array}{ll}
\mathcal{R}_{k}\left(x_{1}\right) a_{k, j}=0 & \text { if } q+j<0, \\
\mathcal{R}_{k}\left(x_{1}\right) a_{k, j}=b_{k, q+j} & \text { if } 0 \leq q+j \leq q .
\end{array}
$$

One can introduce polynomials $\mathcal{S}_{k}$ in a similar way and compute explicitly $\mathcal{S}_{k}\left(e^{x_{1}}\right) a_{k, j}$ for the same values of $j,-2 n \leq j \leq 0$. As a corollary one obtains

Proposition 2.1. Let $f \in E_{n, 1}(\mathbb{K})$ and $k \in \mathbb{N}$, there exist non-zero polynomials $\mathcal{R}_{k}, \mathcal{S}_{k}$ of a single variable, with coefficients in $\mathbb{K}, N_{k} \in \mathbb{N}$, and positive constants $C_{k}, D_{k}$ such that the distributions $a_{k, j},-2 n \leq j \leq 0$, defined by the Laurent development

satisfy the estimates

$$
|f|^{2 \lambda}=\sum_{j=-2 n}^{\infty} a_{k, j}(\lambda+k)^{j}
$$

$$
\left|\left\langle\mathcal{R}_{k}\left(x_{1}\right) a_{k, j}, \varphi\right\rangle\right|+\left|\left\langle\mathcal{S}_{k}\left(e^{x_{1}}\right) a_{k, j}, \varphi\right\rangle\right| \leq C_{k}\|\varphi\|_{N_{k}} \max _{x \in \operatorname{supp}(\varphi)} e^{\left(D_{k} \varrho(x)\right)},
$$

where $\varphi \in \mathcal{D}\left(\mathbb{C}^{n}\right), \varrho(x)=\log (1+|x|)+\left|\Re x_{1}\right|$.

Corollary 2.1. If $\mathbb{K} \subseteq \overline{\mathbb{Q}}$, there are integers $m_{k} \in \mathbb{N}$, and two constants $C_{k}^{\prime}, D_{k}^{\prime}>0$ such that the estimate (16) implies

$$
\left|\left\langle x_{1}^{m_{k}} a_{k, j}, \varphi\right\rangle\right| \leq C_{k}^{\prime}\|\varphi\|_{N_{k}} \max _{x \in \operatorname{supp}(\varphi)} e^{\left(D_{k}^{\prime} \varrho(x)\right)}
$$

In reality, one needs these estimates for the distributions involved in the analytic continuation of distribution-valued holomorphic functions of the form $\left|f_{1}\right|^{2 \lambda_{1}} \ldots\left|f_{p}\right|^{2 \lambda_{p}} /\left(\left|f_{1}\right|^{2}+\ldots+\left|f_{p}\right|^{2}\right)^{m}$. These functions have already appeared in our previous work $[2,10]$. The existence of an analytic continuation as a meromorphic function of $\lambda_{1}, \ldots, \lambda_{p}$ follows from Hironaka's resolution of singularities, but since we want to control the distributions that appear as coefficients in the Laurent developments about some pole, that is, we would like to obtain estimates similar to those of Proposition 2.1 and Corollary 2.1, we need to find some kind of functional equation that provides the analytic continuation. Since it is easier to provide functional equations for $\left|f_{1}\right|^{2 \lambda_{1}} \ldots\left|f_{p}\right|^{2 \lambda_{p}}$, we need a technical trick to reduce this kind of quotients of functions to products. It is based on a simple lemma about the inverse Mellin transform. In order to simplify its writing let us introduce the following notation.

For $t_{1}, \ldots, t_{p}>0, \mu_{1}, \ldots, \mu_{p} \in \mathbb{C}$, we let

$$
t^{* \mu}:=t_{1}^{\mu_{1}} \ldots t_{p}^{\mu_{p}} .
$$

Given $s_{1}, \ldots, s_{p-1}, \beta \in \mathbb{C}$, let

$$
d s:=d s_{1} \ldots d s_{p-1}, s_{p}:=\beta-s_{1}-\ldots-s_{p-1}, \tilde{\mu}_{j}:=\mu_{j}-s_{j}(1 \leq j \leq p) .
$$

We also let $s:=\left(s_{1}, \ldots, s_{p-1}\right), \tilde{s}:=\left(s_{1}, \ldots, s_{p}\right)$, with $s_{p}$ as previously defined. 
Recall also the somewhat standard notation,

$$
\Gamma[a]:=\Gamma\left[a_{1}, \ldots, a_{k}\right]:=\Gamma\left(a_{1}\right) \ldots \Gamma\left(a_{k}\right),
$$

for complex values $a_{j}$ such that the Euler Gamma function is defined. Finally, as long as there is no possibility of confusion, we shall use the following abbreviated notation for multiple integrals on lines parallel to the imaginary axes. Let $\gamma=$ $\left(\gamma_{1}, \ldots, \gamma_{p-1}\right)$ be a vector of real components, then, for any integrable function $F$,

$$
\int_{\gamma-i \infty}^{\gamma+i \infty} F(s) d s:=\int_{\gamma_{1}-i \infty}^{\gamma_{1}+i \infty} \cdots \int_{\gamma_{p-1}-i \infty}^{\gamma_{p-1}+i \infty} F(s) d s_{1} \ldots d s_{p-1}
$$

Lemma 2.2. Let $t_{1}, \ldots, t_{p}>0, \mu_{1}, \ldots, \mu_{p} \in \mathbb{C}, \Re \beta>1, P \in \mathbb{C}\left[\mu_{1}, \ldots, \mu_{p}\right]$, then, with the previous notation,

$$
P(\mu) \frac{t^{* \mu}}{\left(t_{1}+\ldots+t_{p}\right)^{\beta}}=\frac{1}{(2 \pi i)^{p-1} \Gamma(\beta)} \int_{\gamma-i \infty}^{\gamma+i \infty} \Gamma[\tilde{s}] P(\tilde{\mu}) t^{* \tilde{\mu}} d s
$$

for any $\gamma_{j}>0$ such that $\gamma_{1}+\ldots+\gamma_{p-1}<\Re \beta-1$.

We apply this lemma to the study of the coefficients in the Laurent expansion about $\mu=0$ of the analytic continuation of

$$
\mu \mapsto|f|^{* 2(\mu t-\underline{k})} /\|f\|^{2 m},
$$

where $t \in] 0, \infty\left[^{p}\right.$ is a vector to be chosen below, $\mu \in \mathbb{C}, k \in \mathbb{Z}, \underline{k}$ is the $p$-dimensional vector $(k, \ldots, k), m \in \mathbb{N}^{*}, f_{j} \in E_{n, 1}(\mathbb{K}),\|f\|^{2 m}=\left(\left|f_{1}\right|^{2}+\ldots+\left|f_{p}\right|^{2}\right)^{m}$, and, keeping with the previous notation, $|f|^{* r}=\left|f_{1}\right|^{r_{1}} \ldots\left|f_{p}\right|^{r_{p}}$ for any vector $r=\left(r_{1}, \ldots, r_{p}\right)$ (similar meaning for $f^{* r}$ ).

From Proposition 1.2 we conclude that there is a polynomial $A\left(\lambda, x_{1}\right)$ and differential operators $Q_{1, j}\left(\lambda, x, e^{x_{1}}, e^{-x_{1}}, \partial / \partial x\right)$ such that

$$
A\left(\lambda, x_{1}\right) f_{1}^{\lambda_{1}} \ldots f_{p}^{\lambda_{p}}=Q_{1, j}(\lambda)\left(f_{1}^{\lambda_{1}} \ldots f_{j}^{\lambda_{j}+1} \ldots f_{p}^{\lambda_{p}}\right) .
$$

One can iterate this functional equation and restrict our attention to $\lambda=\mu$. After some work one obtains the following result.

Proposition 2.2. Let $f_{1}, \ldots, f_{p} \in E_{n, 1}(\mathbb{K})$, then, for any $\left.t \in\right] 0,1\left[^{p}\right.$ (outside a countable union of $\mathbb{K}$-algebraic hypersurfaces, which depend on the $f_{j}$ ) and any $k \in \mathbb{Z}, m \in \mathbb{N}^{*}$, there are polynomials $\mathcal{R}_{k}$ and $\mathcal{S}_{k}$ in $\mathbb{K}[u]$ and constants $C_{k}, D_{k}>0, N_{k} \in \mathbb{N}$ such that if $a_{k, j} \in \mathcal{D}^{\prime}\left(\mathbb{C}^{n}\right)$ denote the coefficients of the Laurent expansion

$$
\frac{|f|^{* 2(\mu t-\underline{k})}}{\|f\|^{2 m}}=\sum_{j=-2 n}^{\infty} a_{k, j} \mu^{j},
$$

then, for $-2 n \leq j \leq 0, \varphi \in \mathcal{D}\left(\mathbb{C}^{n}\right)$,

$$
\left|\left\langle\mathcal{R}_{k}\left(x_{1}\right) a_{k, j}, \varphi\right\rangle\right|+\left|\left\langle\mathcal{S}_{k}\left(e^{x_{1}}\right) a_{k, j}, \varphi\right\rangle\right| \leq C_{k}\|\varphi\|_{N_{k}} \max _{x \in \operatorname{supp}(\varphi)} e^{\left(D_{k} \varrho(x)\right)},
$$

where $\varrho(x)=\log (1+|x|)+\left|\Re x_{1}\right|$. 
Corollary 2.2. If $\mathbb{K} \subseteq \overline{\mathbb{Q}}$, there is an integer $\nu_{k} \in \mathbb{N}$, and positive constants $C_{k}^{\prime}, D_{k}^{\prime}$ such that

$$
\left|\left\langle x_{1}^{\nu_{k}} a_{k, j}, \varphi\right\rangle\right| \leq C_{k}^{\prime}\|\varphi\|_{N_{k}} \max _{x \in \operatorname{supp}(\varphi)} e^{\left(D_{k}^{\prime} \varrho(x)\right)} .
$$

3. Localization of ideals and applications. In [7] we gave some sufficient conditions, albeit sometimes hard to verify, so that if $f_{1}, \ldots, f_{n}$ are exponential polynomials in $n$ variables with integral frequencies whose variety of common zeros $V=\left\{z \in \mathbb{C}^{n}: f_{1}(z)=\ldots=f_{n}(z)=0\right\}$ is discrete or empty, then the ideal $I$ generated by them in the space $A_{\varrho}\left(\mathbb{C}^{n}\right), \varrho(z)=\log (1+|z|)+|\Re z|$ coincides with $I_{\text {loc }}$ the ideal of those functions in $A_{\varrho}\left(\mathbb{C}^{n}\right)$ which can locally be obtained as linear combinations of the $f_{j}$ with holomorphic coefficients. In particular, $I$ is closed and localizable (i.e., $I=\bar{I}=I_{\text {loc }}$ ). In fact, the conditions given in [7] implied that the $n$-tuple $f_{1}, \ldots, f_{n}$ was slowly decreasing in the sense of [4]. This has a certain number of interesting consequences for the harmonic analysis of the solutions of the system of difference-differential equations in $\mathbb{R}^{n}$ with symbol given by the $f_{j}$. In [6] we had proved that in case $n=2$, the discreteness of $V$ was enough to ensure that the pair $f_{1}, f_{2}$ is slowly decreasing. This led to the conjecture in [7] that if the coefficients of the $f_{j}$ are algebraic numbers, the discreteness of $V$ should be enough to prove that $f_{1}, \ldots, f_{n}$ is slowly decreasing or, at least, that $I$ is closed and localizable. Examples were given showing that this last statement could fail if the algebraicity of the coefficients was not true. On the other hand, we show in this section that if $f_{1}, \ldots, f_{p} \in E_{n, 1}(\mathbb{C})$ define a complete intersection variety, that is $\operatorname{dim} V \leq n-p$, then $I$ is closed and, moreover, $I=I_{\text {loc }}$. In the case $V$ is not a complete intersection we show that the local algebraic closure $\hat{I}$ and the radical $\sqrt{I}$ are closed. That is, these theorems are valid without any restrictions on the coefficients, whereas to extend them to exponential polynomials with two main frequencies one needs to impose arithmetic restrictions both on the frequencies and the coefficients.

The first harmonic analysis result that follows from the statements of the previous section is a localization theorem. The proof depends on the use of HenkinAndersson-Berndtsson type integral representations [15] and analytic continuation of powers as proposed in [2].

Theorem 3.1. Let $f_{1}, \ldots, f_{p} \in E_{n, 1}(\mathbb{C})$ define a complete intersection variety $V$. The ideal I generated by them in $A_{\varrho}\left(\mathbb{C}^{n}\right), \varrho(z)=\log (1+|z|)+\left|\Re z_{1}\right|$ is localizable.

What can one say when we do not assume the ideal is either complete intersection or its variety is discrete? There are several ideals containing $I=I\left(f_{1}, \ldots, f_{p}\right)$. First, let us recall that $\sqrt{I}$, the radical of $I$, is the set of all elements $F \in A_{\varrho}\left(\mathbb{C}^{n}\right)$ such that $F^{k} \in I$ for some $k \in \mathbb{N}$. Second, let $\hat{I}$, the local integral closure of $I$, be the set of all elements $F \in A_{\varrho}\left(\mathbb{C}^{n}\right)$ such that for every point $x_{0} \in \mathbb{C}^{n}$ there is 
a neighborhood $U$ and a constant $C_{x_{0}}>0$ such that

$$
|F(x)| \leq C_{x_{0}}\|f(x)\|=C_{x_{0}}\left(\sum_{j=1}^{p}\left|f_{j}(x)\right|^{2}\right)^{1 / 2}, \forall x \in U .
$$

For $W$ open in $\mathbb{C}^{n}$, let $I_{W}$ denote the ideal generated by $f_{1}, \ldots, f_{p}$ in $\mathcal{H}(W)$. It follows from [22] that $F \in \hat{I}$ if and only if for every $x_{0} \in \mathbb{C}^{n}$ there is an open neighborhood $W$, a positive integer $N$, and functions $\varphi_{1}, \ldots, \varphi_{N}$ such that

$$
F^{N}+\varphi_{1} F^{N-1}+\ldots+\varphi_{N}=0 \text { in } W, \quad \text { and } \varphi_{j} \in I_{W}^{j} .
$$

Finally, let $I(V)=\left\{F \in A_{\varrho}\left(\mathbb{C}^{n}\right): F \mid V=0\right\}$. Note that for a function $F$ to belong to $I_{\text {loc }}$ means that it vanishes on the points of the variety $V$ with some multiplicity, whereas in $I(V)$ the common multiplicities of $f_{1}, \ldots, f_{p}$ are disregarded. It is obvious that $I(V)$ is a closed ideal, and we recall that the same is true for $I_{\text {loc. }}$. Some inclusions between these ideals are clear:

$$
I \subseteq I_{\text {loc }} \subseteq \hat{I} \subseteq I(V), \quad \sqrt{I} \subseteq I(V) .
$$

It is also clear that, in general, we do not have $I_{\text {loc }}=I(V)$. We are now ready to state two important results.

TheOrem 3.2. Let $I$ be the ideal in $A_{\varrho}\left(\mathbb{C}^{n}\right)$ generated by $f_{1}, \ldots, f_{p} \in E_{n, 1}(\mathbb{C})$, $V=\left\{x \in \mathbb{C}^{n}: f_{1}(x)=\ldots=f_{p}(x)=0\right\}$. Then $\sqrt{I}=I(V)$.

TheOREm 3.3. Let $I$ be the ideal of the previous theorem and let $m$ be given by $m=\inf (p+1, n)$, then $\hat{I}^{2 m} \subseteq I$.

One of the remarkable consequences of Theorem 3.3 is the conclusion that sometimes the variety of common zeros is interpolating (see [4, 5] for background information on this question.)

Proposition 3.1. Let $f_{1}, \ldots, f_{n} \in E_{n, 1}(\mathbb{C})$ be such that $\operatorname{dim} V=0$ and $J(x) \neq$ 0 for every $x \in V$, where $J$ is the Jacobian determinant of the $f_{j}$. Then there is a constant $C>0$ such that

$$
|J(x)| \geq \exp \left(-C\left(\left|\Re x_{1}\right|+\log (2+|x|)\right)\right) \quad \forall x \in V .
$$

Thus, $V$ is an interpolating variety in the space $A_{\sigma}\left(\mathbb{C}^{n}\right)$ for any weight $\sigma \geq$ $\left|\Re x_{1}\right|+\log (2+|x|)$.

In fact, one has a stronger result. Let $f_{1}, \ldots, f_{p} \in E_{n, 1}(\mathbb{C})$ be such that $\operatorname{dim} V=k$ and assume that, at every point $x \in V$, there is a $k \times k$ minor of the Jacobian matrix $D f$ of $f_{1}, \ldots, f_{p}$, which does not vanish. Then, the variety $V$ is an interpolation variety for any weight $\geq\left|\Re x_{1}\right|+\log (2+|x|)$. Namely, if we let $J_{1}, \ldots, J_{l}$ denote all the $k \times k$ minors of $D f$, then

$$
\left|J_{1}(x)\right|+\ldots+\left|J_{l}(x)\right| \geq \exp \left(-C\left(\left|\Re x_{1}\right|+\log (2+|x|)\right)\right) .
$$

From [5, Theorem 1], one obtains that $V$ is an interpolating variety.

We conclude this short summary of some of our recent results with an indication of some simple applications to harmonic analysis that can be obtained from 
the above statements and the methods of [4]. For that purpose, let us recall that a linear differential operator $P(D)$ with constant coefficients and commensurable time lags is a finite sum of the form

$$
(P(D) \varphi)(t, x)=\sum p_{j k}\left(D^{j} \varphi\right)(t-k T, x),
$$

$t \in \mathbb{R}, x \in \mathbb{R}^{n},(n \geq 0), D=\left(\partial / \partial t, \partial / \partial x_{1}, \ldots, \partial / \partial x_{n}\right), j \in \mathbb{N}^{n+1}, k \in \mathbb{Z}, T>0$, and $p_{j k} \in \mathbb{C}$. The symbol of this operator $P(\tau, \xi)$ is the element of $E_{n+1,1}(\mathbb{C})$ given by

$$
P(\tau, \xi):=e^{i(t \tau+x \cdot \xi)} P(D) e^{-i(t \tau+x \cdot \xi)}=\sum p_{j k}(-i \zeta)^{j} e^{i k T \tau},
$$

with $\zeta=(\tau, \xi)$. (By the introduction of the new coordinate $\xi_{0}=i T \tau$, we are in the case of exponential polynomials considered at the beginning of this section.)

TheOREM 3.4. Let $P_{1}(D), \ldots, P_{n+1}(D)$ be differential operators with time lags as in (20), with the property that the characteristic variety

$$
V:=\left\{\zeta \in \mathbb{C}^{n+1}: P_{l}(\zeta)=0,1 \leq l \leq n+1\right\}
$$

is discrete and all the points of $V$ are simple. Then, every solution $\varphi \in \mathcal{E}\left(\mathbb{R}^{n+1}\right)$ (resp., $\varphi \in \mathcal{D}^{\prime}\left(\mathbb{R}^{n+1}\right)$ ) of the overdetermined system

$$
P_{1}(D) \varphi=\ldots=P_{n+1}(D) \varphi=0
$$

can be represented in a unique way in the form of a series of exponential solutions of the system (22), namely,

$$
\varphi(t, x)=\sum_{\zeta \in V} c_{\zeta} e^{i(t \tau+x \cdot \xi)} .
$$

This series is convergent in the topology of $\mathcal{E}\left(\mathbb{R}^{n+1}\right)\left(\right.$ resp., $\left.\mathcal{D}^{\prime}\left(\mathbb{R}^{n+1}\right)\right)$.

\section{References}

[1] M. F. Atiyah, Resolution of singularities and division of distributions, Comm. Pure Appl. Math. 23 (1970), 145-150.

[2] C. A. Berenstein, R. Gay and A. Yger, Analytic continuation of currents and division problems, Forum Math. 1 (1989), 15-51.

[3] C. A. Berenstein, R. Gay, A. Vidras and A. Yger, Residues and Bézout Identities, Progr. Math. 113, Birkhäuser, 1993.

[4] C. A. Berenstein and B. A. Taylor, Interpolation problems in $\mathbb{C}^{n}$ with applications to harmonic analysis, J. Analyse Math. 37 (1980), 188-254.

[5] - - - On the geometry of interpolating varieties, in: Séminaire Lelong-Skoda 1980-81, Springer, Heidelberg, 1-25.

[6] C. A. Berenstein, B. A. Taylor et A. Yger, Sur les systèmes d'équations différencedifférentielles, Ann. Inst. Fourier (Grenoble) 33 (1983), 109-130.

[7] C. A. Berenstein and A. Yger, Ideals generated by exponential polynomials, Adv. in Math. 60 (1986), 1-80.

[8] _, - On Eojasiewicz type inequalities for exponential polynomials, J. Math. Anal. Appl. 129 (1988), 166-195. 
[9] C. A. Berenstein and A. Yger, Analytic Bézout identities, Adv. in Appl. Math. 10 (1989), 51-74.

[10] -, -, Effective Bézout estimates in $\mathbb{Q}\left[z_{1}, \ldots, z_{n}\right]$, Acta Math. 66 (1991), 69-120.

[11] - - -, Une formule de Jacobi et ses conséquences, Ann. Sci. Ecole Norm. Sup. 24 (1991), 363-377.

[12] - - - About Ehrenpreis' Fundamental Principle, in: Geometric and Algebraic Aspects in Several Complex Variables, C. A. Berenstein and D. C. Struppa (eds.), Editel, Rende, 1991, 47-61.

[13] - - - Formules de représentation intégrale et problèmes de division, in: Diophantine Approximations and Transcendental Numbers, P. Philippon (ed.), Walter de Gruyter, Berlin, 1992, 15-37.

[14] -, -, D-modules and exponential polynomials, Compositio Math. (1995), to appear.

[15] B. Berndtsson and M. Andersson, Henkin-Ramirez formulas with weight factors, Ann. Inst. Fourier (Grenoble) 32 (1982), 91-110.

[16] I. N. Bernstein, The analytic continuation of generalized functions with respect to a parameter, Functional Anal. Appl. 6 (1972), 273-285.

[17] J.-E. Björk, Rings of Differential Operators, North-Holland, Amsterdam, 1979.

[18] A. Borel, Operations on algebraic $\mathcal{D}$-modules, in: Algebraic $\mathcal{D}$-modules, A. Borel (ed.), Academic Press, Boston, 1987, 207-269.

[19] L. Ehrenpreis, Fourier Analysis in Several Complex Variables, Wiley, 1970.

[20] D. I. Gurevich, Counterexamples to a problem of L. Schwartz, Functional Anal. Appl. 9 (1975), 116-120.

[21] L. Hörmander, The Analysis of Linear Partial Differential Operators, II, Springer, Berlin, 1983.

[22] M. Lejeune et B. Teissier, Quelques calculs utiles pour la résolution des singularités, Séminaire École Polytechnique, 1972, 130.

[23] B. Lichtin, Generalized Dirichlet series and B-functions, Compositio Math. 65 (1988), $81-120$.

[24] L. Schwartz, Théorie générale des fonctions moyenne-périodiques, Ann. of Math. 48 (1947), 857-929.

[25] C. Sabbah, Proximité évanescente II. Equations fonctionnelles pour plusieurs fonctions analytiques, Compositio Math. 64 (1987), 213-241. 